\title{
SŁAWOMIR SOCZYŃSKI \\ Menschenwürde als sittlicher Faktor im Personalmanagement jeder Organisation
}

Der Begriff Organisation ist sehr umfassend und wird auch sehr unterschiedlich verstanden. Er gründet auf dem griechischen Wort órganon „Werkzeug" - und kann als Spezialteil, das einer Aufgabe in der Gesamtheit dient, verstanden werden ${ }^{1}$. In der Wissenschaft wird Organisation als Funktion, als Instrument oder Institution verstanden. Für unsere Analyse wird die letzte Bedeutung nämlich Organisation als Institution - relevant und zwar Institution als eine Gruppe von Menschen, die zusammen, systematisch, koordiniert und geordnet arbeitet, um ein Ziel zu erreichen.

Schon eine oberflächliche Beobachtung unserer Gesellschaft lässt uns feststellen, dass sie voll von solch definierten Organisationen ist; als Beispiele können wir alle Unternehmen, Bundes- oder Feuerwehr, Krankenhäuser, Schulen, religiöse Organisationen usw. anführen. Die meisten von uns sind auch Mitglieder solcher Organisationen. In ihnen sind wir geboren und assoziiert, in ihnen arbeiten wir und genießen wir unsere Freizeit. Ohne Organisationen ist unsere Gesellschaft heute nur schwer vorstellbar. Sie sind ein wesentlicher Bestandteil der Gesellschaft geworden, der vor allem den Menschen dienen soll.

Dabei müssen wir uns im Klaren sein, dass wir es hier mit keinem Automatismus zu tun haben. Institutionen, in sich und als solche, sind wirkungslos. Menschen mit ihren Zielen und Wertsystemen, ihren Qualifikationen und Motivationen entscheiden darüber, ob die Organisation ihre Ziele erreicht oder nicht. Die

Sławomir S O C Z Y Ń S K I SDS, ks. dr, pracownik Instytutu Dziennikarstwa i Komunikacji Społecznej na Wydziale Nauk Społecznych Uniwersytetu Papieskiego Jana Pawła II w Krakowie, e-mail: slawomir.soczynski@upip2.edu.pl

\footnotetext{
${ }^{1}$ M. B i e 1 s k i: Organizacje - istota, struktury, procesy. Łódź 1997 S. 68.
} 
persönliche Verantwortung eines jeden Mitglieds ist dabei gefragt, wobei die Führungskräfte eine besondere Rolle zu erfüllen haben. Die Kompetenz des Managements und dessen Führungsstil entscheiden oft über den effektiven Einsatz der Ressourcen und den Motivationsgrad der Mitarbeiter.

In den Lehrbüchern und Fachzeitschriften finden wir eine ganze Reihe von Theorien zum Thema Führung und Personalführung. Viele von diesen reklamieren einen hohen Neuigkeitsgrad für sich. Die wichtigste Ursache ihrer Entstehung und Entwicklung ist die Veränderung des sozialökonomischen Umfeldes, in dem die Organisationen agieren. Die technologischen Innovationen, der Globalisierungsprozess in vielen Bereichen unseres Lebens, aber auch demografische Probleme sowie der Wertenwandel zwingen zum Umdenken und Umstellen in allen Bereichen einer Organisation. Sowohl ihre strategischen Ziele, ihre Ressourcen als auch ihre Führungsmethoden müssen durchgedacht werden.

Bei der Suche nach neuen Führungsmodellen sind Effektivität und Gewinnmaximierung wichtige Motive, aber nicht die einzigen. Wichtig sind auch die Werte wie: Wissen, Kompetenz, Kreativität und Ethik.

Interessiert daran sind nicht nur die wirtschaftlich orientierten Unternehmen. Auch die Organisationen, non-profit' brauchen neue Methoden, mit denen sie ihre Probleme besser und effektiver managen können. Der Unterschied im Managen dieser beiden Gruppen, zumindest in manchen Bereichen, ist auch nicht so groß, wie man meinen könnte. Peter Drucker behauptet, dass nur 10\% der Angelegenheiten, die eine Organisation zu managen hat, etwas mit ihrer Mission, ihrer spezifischen Kultur, ihrer Historie und Begrifflichkeit zu tun hat; 90\% sind dagegen allgemeiner $\mathrm{Art}^{2}$.

Selbstverständlich kann man mit Drucker streiten und auch relativ leicht belegen, dass es doch größere Unterschiede zwischen den Organisationen gibt. A.B. Albarran weist zum Beispiel darauf hin, dass Medienunternehmen wegen ihres außergewöhnlichen Charakters und ihrer Mission in der Gesellschaft gehindert sind, die allgemeinen Theorien des Managements zu verwenden. Dies gilt sicher auch für religiöse Organisationen, die weniger auf die Veränderungen der Umgebung schauen, mehr der Tradition verbunden sind und wohl auch stärker auf die universalen Werte achten, so dass sie in ihrem Führungsstil andere Aspekte und Werte berücksichtigen.

Gewiss könnte man noch mehr Beispiele nennen, die gegen die Behauptung Peter Druckers sprechen. Für unsere Überlegungen aber haben die Unterschiede zwischen spezifischen Organisationen jedoch keine entscheidende Bedeutung. Es geht uns doch um das Personalmanagement auf der ethischen Ebene. Die Ethik ist für uns insofern interessant, als dass der Führungsstil in allen Organisationen

\footnotetext{
${ }^{2}$ P. D r u c k e r: Management Challenges for the 21st Century. New York 2009 S. 8
} 
gleich und verantwortungsvoll sein sollte und als Bewertungsgrundlage einen universalen Charakter hat.

\section{Menschenwürde als Maßstab}

Der Begriff Organisationsführung wird als sehr capacious gesehen und bedeutet nicht nur, mit Hilfe von humanen, finanziellen, organisatorischen, physischen und technologischen Ressourcen die Organisation zum Ziel zu führen, sondern auch strategische Ziele zu bestimmen, die mit der Mission und Vision einer Organisation übereinstimmen. Diese Planung und ihre Durchführung soll in erster Linie der Organisation dienen, besser gesagt, den Erwartungen, die die Gesellschaft ihr gegenüber hat. Dabei ist unwichtig, ob sie materieller, kultureller oder anderer Natur sind. Jede Organisation, die ihre Aufgabe nicht erfüllen kann, erübrigt sich. Die neuen Führungsmethoden zielen darauf, genau das zu verhindern. Es gilt also, die Effektivität zu steigern, um die einzelnen Bereiche der Organisation zu entwickeln, unter diesen auch den Bereich der Personalführung.

Organisationsmanagement kann selbstverständlich nicht utopisch sein; es muss sich an den Marktregeln orientieren und sollte dabei menschenfreundlich sein, anders gesagt, ethisch sein. Die Normen von Markt und Moral sollten berücksichtigt werden. Es ist wahr, dass es in der gegenwärtigen, pluralistischen Gesellschaft immer schwerer fällt, eine gemeinsame „Ebene der Werte“ zu finden. Es scheint aber auch, dass trotz aller unterschiedlichen Weltanschauungen und Wertesysteme, eine Mehrheit der Menschen das Menschsein und die Menschenwürde als einen grundlegenden Wert akzeptiert. Das Bewusstsein für die eigene Würde, das die Menschen in der Neuzeit gewonnen haben, vor allem die arbeitenden Menschen, Männer und Frauen, hat universelle Dimensionen. Dieses Phänomen wurde in der Geschichte nicht nur durch eine fortschreitende Verkündigung und Verteidigung der Menschenrechte deutlich gemacht, sondern auch in der tiefen Sehnsucht nach einer lebendigen und sinnvoll sozialen Gerechtigkeit $^{3}$. Diese Würde, wie Josef Seifert schreibt, hat zum einen einen von aller Willkür unabhängigen objektiven Wert, b) zum anderen einen erhabenen Wert, der keinen Preis hat und nicht veräußerlich ist, und c) schließlich einen Gegenstand moralischer Forderungen und die Quelle von Menschenrechten ${ }^{4}$. Die Menschenwürde ist also auch die Quelle der Grundrechte des Menschen, nämlich: das Recht zu leben und nicht als reines Mittel gebraucht zu werden. Der Mensch ist immer als ein Subjekt zu betrachten, der ein eigenes Wertesystem, eigene Bedürfnisse und eigene Verpflichtungen hat.

\footnotetext{
${ }^{3}$ J a n P a w e 1 I I: Do robotników Solvay'u. Dokumenty społecznej nauki Kościoła. RzymLublin 1987.

${ }^{4}$ J. S e i f e r t: Dimensionen und Quellen der Menschenwürde S. 8. In: http://homepage.ruhruni-bochum.de/walter.schweidler/seifert4.doc. (abgerufen am 2.04.2015)
} 
Als freies und rationales Wesen sollen der Mensch und sein Werden in der Mitte allen menschlichen Handelns stehen. Es geht - wie ein zeitgenössischer Philosoph gesagt und auch das Konzil festgestellt hat - nicht so sehr darum, »mehr zu haben", sondern »mehr zu sein«. In der Tat besteht schon eine wirkliche, erkennbare Gefahr, dass der Mensch bei dem enormen Fortschritt in der Beherrschung der gegenständlichen Welt die entscheidenden Fäden, durch die er sie beherrscht, aus der Hand verliert und ihnen auf verschiedene Weise sein Menschsein unterordnet und selbst Objekt wird von vielfältigen, wenn auch oft nicht direkt wahrnehmbaren Manipulationen durch die Organisation des gesellschaftlichen Lebens, durch das Produktionssystem und durch den Druck der sozialen Kommunikationsmittel. Der Mensch kann weder auf sich selber verzichten, noch auf den Platz, der ihm in der sichtbaren Welt zukommt; er darf nicht Sklave der Dinge, Sklave der Wirtschaftssysteme, Sklave der Produktion, Sklave der eigenen Produkte werden ${ }^{5}$.

Der Mensch muss also der Ausgangs- und Endpunkt aller Handlungen einer Organisation sein, was in seiner Würde begründet ist. Die ontologische Personenwürde, ist "unverfügbar", insofern kein Mensch das Recht hat, über den Menschen in seiner Würde zu verfügen wie über Tiere oder seinen Besitz, und "unabdingbar", da sie in notwendiger und unanfechtbarer Weise zur Person gehört ${ }^{6}$. So lässt sich der Bezugspunkt der Verantwortung im Personalmanagement wie folgt beschreiben: Es ist der Mensch mit seiner Würde sowie die daraus abgeleiteten Werte wie Meinungsfreiheit, Gemeinwohl, Gerechtigkeit und Solidarität.

Wenn die neuen Konzeptionen ethisch und menschenwürdig werden wollen, sollte bei ihrer Entwicklung und Verwirklichung berücksichtig werden, dass nicht nur materielle Gründe relevant sind, sondern auch die Berücksichtigung der gesellschaftlichen Erwartungen und das Wohl aller Interessenten der Organisation.

\section{Personalmanagement als unterstützende Aufgabe}

Personalmanagement ist der Teil des Organisationsmanagements, der sich mit dem Faktor Arbeit und dem Faktor Personal auseinandersetzt. Seine Kernaufgaben sind die Bereitstellung und der zielorientierte Einsatz von Personal ${ }^{7}$. In den letzten Jahren hat sich das Denken über die Rolle der Mitglieder einer Organisation stark verändert. Besonders sichtbar ist das bei den Unternehmern. Arbeiter werden nicht mehr bloß als „Erfüllungswerkzeuge“, sondern als wichtigster

\footnotetext{
${ }^{5}$ J o h a n nes P a u 1 I I: Enzyklika Redemptor hominis 1979.

${ }^{6} \mathrm{~J}$. S e i f e rt: Dimensionen und Quellen der Menschenwürde. op. cit. s. 16-17.

${ }^{7}$ E. G a u g l e r, W. A. O e c h s l e r, W. W e b e r: Personalwesen. In: Handwörterbuch des Personalwesen. Hrsg. E. G a u g l e r, W.A. O e c h s l e r, W. W e b e r. Stuttgart ${ }^{3} 2004$ Sp. 1653.
} 
Teil der Organisation betrachtet. Zwar ist dieses Umdenken oft nicht, wie man sich das wünschen würde, durch die Achtung der Menschenwürde motiviert, sondern durch die Erkenntnis, dass die Arbeiter der entscheidende Faktor des Erfolges sind, trotzdem sollte man wahrnehmen, dass der Umgang mit den Menschen heute, dank dieses Umdenkens, mindestens in vielen Organisationen den hochentwickelten Ländern, menschlicher geworden ist.

Dadurch hat sich auch der Aufgabenbereich des Personalmanagements verändert. Heute geht es um viel mehr als nur um Personalverwaltung, wie es in der Vergangenheit oft war. Gabler definiert Personalmanagement als die Summe der mitarbeiterbezogenen Gestaltungsmaßnahmen zur Verwirklichung der strategischen Unternehmensziele ${ }^{8}$. Zu den Aufgaben des Personalmanagements gehören also: Personalplanung, -entwicklung, und -führung.

\section{Partizipation der Mitglieder an der Planung}

Die erste Aufgabe des Personalmanagements ist Personalplanung. Weil der Mensch eine Person ist, d.h. ein subjekthaftes Wesen, das imstande ist, auf geordnete und rationale Weise zu handeln, ist er auch fähig, über sich zu entscheiden, und auf Selbstverwirklichung ausgerichtet ${ }^{9}$. Aus dieser Feststellung ergibt sich die Berechtigung, an den Organisationsplanungen teilnehmen zu können. Der Menschen darf nie bloß als Objekt, sondern muss als Subjekt behandelt werden. Im Blick auf alle Planungen, die mit ihm zu tun haben, sollte er also auch das Recht der Ablehnung bzw. Zustimmung haben. Ihm dieses Mitbestimmungsrecht zu verweigern, ihm nicht zu ermöglichen, sich zu den mit ihm verbundenen Plänen zu äußern, würde bedeuten, ihn zu einem Mittel zum Zweck zu reduzieren. Eine solche Instrumentalisierung des Menschen hat mit Menschenwürde nichts mehr zu tun.

Der Mensch, der als Objekt behandelt wird, wird seiner Möglichkeit beraubt, am Lauf der Welt und seines Lebens aktiv teilzunehmen, obwohl er das Recht hat, Gestalter seiner Umwelt und ihrer Transformation zu sein. Er wäre bestimmt und nicht der Bestimmende, entschieden und nicht der Entscheidende, initiiert, aber nicht der Initiierende, immer passiv, nie der aus von sich selbst Handelnde ${ }^{10}$.

Im engen Sinn kann man eine Organisation als eine Institution betrachten, die bestimmte, für sie geplante Ziele zu erreichen hat. Sie ist aber darüber hinaus eine Möglichkeit der menschlichen Selbstverwirklichung. Dank seines Engagements in verschiedenen Organisationen kann der Mensch sich entwickeln und

8 http://wirtschaftslexikon.gabler.de/Definition/personalmanagement.html. (abgerufen am 4.04.2015)

${ }^{9}$ J o hannes Paul I I: Enzyklika Laborem exercens 1982

${ }^{10}$ J. L i p i e c: Wolność i podmiotowość człowieka. Kraków 1997 S. 13. 
selbstverwirklichen. Die Zulassung einer Diskussion, vor allem über Vision und Mission der Organisation, aber auch über Ressourcen, Politik und Betriebserfahrung, wird letztlich mehr sein als nur die Gewährung bzw. Wahrnehmung eines Rechtes. Sie führt alle Beteiligten zu einem besseren Verständnis der strategischen Organisationsziele; sie hilft, diese als die Eigenen zu betrachten und sich für sie verantwortlich zu fühlen. Dadurch entsteht die innere Verbundenheit mit der Institution als Gemeinwohl, was letztlich auch der Organisation zu Gute kommt. Das Gefühl der privaten Verantwortung erzeugt nämlich das Bedürfnis, zu dienen ${ }^{11}$.

Im Personalwesen sind neben den fachlichen Kompetenzen, die die Modelle der Personalführung betonen, auch menschliche Kompetenzen wichtig wie: Ehrlichkeit und Respekt, Wahrhaftigkeit und Zuverlässigkeit, Empathie und Verantwortung. Auf diesen Eigenschaften kann eine ethische Kultur der Organisation wachsen, die für die Organisation und ihre Mitglieder von großer Bedeutung ist.

\section{Personalentwicklung}

Wie bereits angesprochen, sollen die Aktivitäten des Personalmanagements auf der Basis der Menschenwürde nicht nur das Organisationsziel, sondern auch die Erwartungen des Personals und das Gemeinwohl berücksichtigen. Wichtig ist dabei eine gute Planung, welche Stelle in der Organisation welche Kompetenzen benötigt. Die Brücke zwischen der Strategie und ihrer Einführung bilden die "Arbeitswertschätzungsprozesse». In diesem Bereich werden Qualifikationsminimum für einen bestimmten Arbeitsplatz, das die Bedingungen der Implementierung der Strategie und der sich daraus ergebenden Kriterien zur Auswahl der Arbeitnehmer für bestimmte Aufgaben berücksichtigt sowie Kriterien für die Bewertung der Arbeitsergebnisse wie auch der Arbeitsqualität definiert ${ }^{12}$. Fehlerhafte Arbeitswertschätzungsprozesse können zu großen Problemen für die Organisation und für die Arbeitsnehmer führen.

Die Aufgabe des Personalmanagements ist vor allem, die Einbeziehung aller Mitarbeiter, mit ihrer Erfahrung und Kompetenz, in die Arbeit der Organisation, um die Organisationsziele so gut, wie es nur möglich ist, zu realisieren, aber auch um den Mitgliedern bei der Entwicklung ihrer eigenen Qualifikationen und bei der Realisierung ihrer persönlichen Ziele zu helfen. Weil immer öfter die Aufgabenerledigung in Arbeitsgruppen geschieht, begründet sich ein positives Ergebnis in der Gruppenbildung wie in der kompetenten Aufgabenverteilung; letztere sollte die Fähigkeiten jedes einzelnen Mitarbeiters beachten. Entsprechend bedeutend sind gute Kenntnisse über die Bedingungen der Organisation, aber auch über

\footnotetext{
${ }^{11} \mathrm{Vgl}$ J. T i s c h n e r: Polska jest Ojczyzna. 1985 S. 126.

12 Z. G o m u ł k a: Motywowanie w praktyce zarzadzania rozwojem ludzi i organizacji. In: „Management and Business Administration. Central Europe”. R. 115 : 2012 Nr. 2 S. 66.
} 
die Mitarbeiter, ihr intellektuelles und emotionales Potenzial und ihre Kommunikationsfähigkeit.

Die Aufgabe einer Organisation ist nicht nur die Verwirklichung ihrer definierten Ziele, sondern auch allen mit ihr verbundenen Subjekten einen Raum der menschlichen Selbstverwirklichung zu bieten. Die Arbeit ist ein Gut für den Menschen - für sein Menschsein - weil er durch die Arbeit nicht nur die Natur umwandelt und seinen Bedürfnissen anpasst, sondern auch sich selbst als Mensch verwirklicht, ja gewissermaßen »mehr Mensch wird « ... ${ }^{13}$. Bei der Arbeit lernt der Mensch seine Aufgaben, entwickelt seine Fähigkeiten, ist bereit, etwas zu wagen, und gewinnt schließlich an Selbstvertrauen.

Arbeit soll also auch der Entwicklung von Fachkompetenz sowie der Persönlichkeitsmerkmale dienlich sein. Ein gerechter Lohn sollte darüber hinaus auch dazu dienen, den Arbeitenden und ihren Familie ein menschenwürdiges Leben zu gewährleisten. Die Familie einen der wichtigsten Bezugspunkte für den rechten Aufbau einer sozial-ethischen Ordnung der menschlichen Arbeit bildet. (...) Ist doch die Familie eine durch die Arbeit ermöglichte Gemeinschaft und die erste, häusliche Schule der Arbeit für jeden Menschen ${ }^{14}$. In der Familie lernt der Mensch, was Gehorsam, Hierarchie, Verantwortung, Zusammenarbeit, aber auch was Freundschaft, Großzügigkeit oder Engagement bedeuten, Kompetenzen also, die später für und in der Organisation nützlich werden.

\section{Personalführung}

In der Literatur wird es heute oft betont, dass Humankapital für moderne Organisationen die wichtigste Ressource ist und dass es der einzige Differenzierungsfaktor im globalen Wettbewerb geworden ist. Unter dem Begriff Humankapital, das im Jahre 2004 mit der Begründung, ,es verletze die Würde des Menschen“, zum Unwort des Jahres ${ }^{15}$ gewählt worden ist, wird „die Summe der Erfahrungen, Fähigkeiten und Fertigkeiten eines Individuums, einer Gruppe, oder der Erwerbsbevölkerung einer Volkswirtschaft, welche im Produktionsprozess aktiv eingesetzt werden kann “16, verstanden, d.h. dass unter Humankapital nicht die Mitarbeiter und Mitarbeiterinnen selbst, sondern nur deren Kompetenzen gemeint sind.

Dabei sollte man sich aber auch bewusst machen, dass die typisch menschlichen Kompetenzen wie Erfahrungen, Fähigkeiten und Fertigkeiten usw. nur einer

${ }^{13}$ J o han nes Paul I I: Enzyklika Laborem exercens. 1982.

${ }^{14}$ Daselbst.

${ }^{15}$ Das „Unwort“ des Jahres wurde von der Jury der sprachkritische Aktion „Unwort des Jahres“ gewählt und soll in erster Linie als Anregung zu mehr sprachkritischer Reflexion dienen. Vgl. Grundsätze. In: unwortdesjahres.net (abgerufen am 12.04.2015).

M. V e l t e n: Investitionen in Humankapital am Beispiel von Kleinunternehmen des Metallsektors in Nicaragua. Freiburg (Breisgau) 1998 S. 5. 
Person und nie einer Organisation gehören können. Selbst wenn sie einer Organisation zugeschrieben werden, kann das nur im übertragenen Sinne gemeint sein. Denn nur die Person, die diese Kompetenzen besitzt, kann sie als ein freier und rationaler Mensch umsetzen. Die Aufgabe des Managers ist es also, die Mitarbeiter und Mitarbeiterinnen so zu führen, dass sie ihre Kompetenzen so gut wie möglich einsetzen können.

Der Soziologe Max Weber teilte in seinem Werk ,Wirtschaft und Gesellschaft" die Führungsstile grundsätzlich in 4 traditionellen Klassen: der patriarchalische, der charismatische, der autokratische und der bürokratische Führungsstil. Als Vorbild hat er die Herrschaft Typologie aus anderen Bereichen genommen und in die Wirtschaft übertragen. Ist gestern der Autoritäre oder der sich aus ihm entwickelte Bürokratische Führungsstil gefragt, so spricht man heute gerne von visionären oder charismatischen Führen. Der autoritäre Vorgesetzte, der kraft seiner Legitimationsmacht führte und weil er alles besser weiß allein Entscheidungs- und Anweisungskompetenz besitzt, ist heute nicht mehr gefragt. Die Herausforderungen der modernen Welt führen dazu, dass der Leiter oder Manager heute, nicht so wie früher, die privilegierte Stellung dessen, der alles weiß, annehmen kann, sondern derjenige sein soll, der sucht und mit Hilfe anderer herausfinden will, was das Beste für die Organisation und alle Interessenten ist.

$\mathrm{Ob}$ die richtigen Entscheidungen getroffen werden, hängt oft von der Ethik und der Methode der Zusammenarbeit auf allen Ebenen der Organisation ab. Äußerst wichtig ist dabei, niemanden in die Rolle des passiven Auftragnehmers zu drängen, der so der bewussten Verwirklichung seiner Aufgaben beraubt würde. Es reicht nicht, den Untergeordneten einen Auftrag zu geben, es ist notwendig, diesen zu begründen und genau zu bestimmen. Sowohl der Sinn des Auftrags als auch das $\mathrm{Maß}$ an Selbständigkeit in seiner Ausführung zu erklären, ist die menschenwürdige Vorgehensweise.

Die Personalführung sollte mehr eine dialogische als die direktive Form annehmen. Die Führungskraft beschreibt den Mitarbeitern und Mitarbeiterinnen Zuständigkeiten, gibt ihnen die benötigte Unterstützung und setzt notwendige Grenzen ${ }^{17}$. Dabei muss die menschliche Verschiedenheit berücksichtig werden, so dass jeder Mensch anders ist und anders geführt werden muss, was aber nicht zweierlei Maß bedeuten darf.

\section{Schlussfolgerungen}

Seit den 80-ger Jahren kann man immer lautere Stimmen hören, die mehr Ethik für verschiedene Bereiche unseres Lebens fordern. Viele Organisationen und Berufsgruppen haben daraufhin einen Verhaltenskodex erstellt und immer

\footnotetext{
${ }^{17}$ B. K. S i m e r s o n, M. L. V e n n: Menedżer jako lider. Warszawa 2010 S. 26.
} 
wieder neue Kodizes entstehen. Trotz dieser Tendenz haben wir aber damit zu tun, dass der Gewinn weiterhin wichtiger ist als der Mensch und die Ethik. Management-Praktiken in den Organisationen, die unethisch sind und die Menschenwürde missachten, wie die Konzentration auf das Ergebnis, nicht auf die soziale Verantwortung, Zwang zur unverhältnismäßigen Anstrengung, Vorrang des Managements vor anderen Interessengruppen und nicht zuletzt die direktive Machtausübung führen zur Unzufriedenheit der Betroffenen und zerstören das Arbeitsklima, was die Erfüllung der Organisationsaufgabe gefährdet. Personalführung, die die Menschenwürde beachtet, gibt den Betroffenen dagegen das Gefühl, ein wichtiger Teil der Organisation zu sein, was wiederum sie zu mehr Engagement motiviert.

\section{Bibliografia}

Bielski M.: Organizacje - istota, struktury, procesy. Łódź 1997.

Drucker P.: Management Challenges for the 21st Century. New York 2009.

Gaugler E.: Oechsler W.A., Weber W.: Personalwesen. W: Handwörterbuch des Personalwesen. Red. E. Gaugler, W.A. Oechsler, W. Weber. Stuttgart ${ }^{3} 2004$.

Gomułka Z.: Motywowanie w praktyce zarzadzania rozwojem ludzi i organizacji. W: „Management and Business Administration. Central Europe” R. 115: 2012 nr 2 s. 63-71. Jan Paweł II: Encyklika Redemptor hominis 1979.

Jan Paweł II: Enzyklika Laborem exercens 1982.

Jan Paweł II: Do robotników Solvay'u. Dokumenty społecznej nauki Kościoła. RzymLublin 1987.

Seifert J: Dimensionen und Quellen der Menschenwürde. W: http://homepage.ruhr-unibochum.de/walter.schweidler/seifert4.doc.

Lipiec J.: Wolność i podmiotowość czlowieka. Kraków 1997.

Simerson B.K., Venn M.L.: Menedżer jako lider. Warszawa 2010.

Tischner J.: Polska jest Ojczyzną. Paryż 1985.

Velten M.: Investitionen in Humankapital am Beispiel von Kleinunternehmen des Metallsektors in Nicaragua. Freiburg (Breisgau) 1998.

\section{ABSTRACT}

\section{Human dignity as a moral determinant of human resource management of each organization}

Throughout our life, most of us belong to many organizations, which are intended to help achieve our goals, both individual and collective. Responsibility for the smooth and successful functioning of the organization lies with the members of its management team. The changing socioeconomic conditions result in the search of new management models. Effectiveness and maximization of profit by organizations are important reasons for this search. The purpose of this paper is to draw attention to the need of an ethical component in the search, which should have human dignity as the most important value, and also to show what the recognition of human dignity as the superior value should result in during the different stages of the management process.

Key words: human dignity, management, organization, church.

Słowa kluczowe: godność ludzka, kierownictwo, organizacja, Kościół. 